\title{
Heavy and light spectroscopy near the physical point, part I: Charm and bottom baryons
}

\author{
R. J. Hudspith ${ }^{* a}$, A. Francis ${ }^{a}$, R. Lewis ${ }^{a}$ and K. Maltman ${ }^{b c}$ \\ ${ }^{a}$ Dept. of Physics and Astronomy, York University, Toronto, Ontario, M3J 1P3, Canada \\ ${ }^{b}$ Dept. of Mathematics and Statistics, York University, Toronto, Ontario, M3J 1P3, Canada \\ ${ }^{c}$ CSSM, University of Adelaide, Adelaide SA 5005, Australia \\ E-mail: renwick.james.hudspith@gmail.com
}

\begin{abstract}
We present results of the hadron spectrum using $n_{f}=2+1$ ensembles with pion masses as low as $\approx 164 \mathrm{MeV}$, placing particular emphasis on measurements of the singly and doubly heavy charm and bottom baryons. Using the Tsukuba tuning for relativistic charm and NRQCD for the bottom quarks we perform measurements of both light and heavy mesons as well as $J^{P}=1 / 2^{+}, 3 / 2^{+}$ baryons for all possible flavor combinations. Our subsequent analysis yields masses with an accuracy below the $1 \%$-level and therefore splittings with good statistical precision. All results are extrapolated to the physical pion mass via a tightly controlled, short, chiral extrapolation.
\end{abstract}

34th annual International Symposium on Lattice Field Theory

24-30 July 2016

University of Southampton, UK

\footnotetext{
*Speaker.
} 


\section{Introduction}

In this paper we introduce our measurement strategy for the hadronic spectrum on ensembles generated by PACS-CS [1] and available via the JLDG/ILDG [2]. The aim of this work is to:

1. Check our methodology against the known spectrum and compare with the original ensemble paper.

2. Utilise a well-tuned charm quark description.

3. Retune the NRQCD action for these ensembles.

4. Compare the baryon splittings and masses of [3] with a different fermion description, in particular those of doubly and triply heavy (charm or bottom quarks).

We believe comparison with [3] is particularly important. As of yet, the LHC has not discovered doubly and triply heavy baryons. Previous lattice studies have predicted the masses of these baryons [4, 5], but only a handful with dynamical fermions [6, 7, 3]. It is beneficial to the community as a whole to compare results with different actions and using different methodologies to provide reliable predictions for these quantities. The measurements presented here will be much closer to the chiral limit than those of [3], reducing possible systematics from the chiral extrapolation.

\section{Background}

We use gauge fixed wall sources ${ }^{1}$. These sources provide good statistical reduction at fixed cost with better overlap to the ground state seen as an earlier onset in the effective mass in comparison to point and smeared sources. These sources automatically zero momentum project [9] and are slightly more difficult to use for extracting matrix elements from the resulting amplitudes.

\subsection{Mesons}

Correlation functions are defined as (where $s_{1 / 2}$ is a source-type label),

$$
\begin{aligned}
C_{\mathscr{O}_{1} \mathscr{O}_{2}}^{s_{1} s_{2}}(p, t) & =\left\langle\sum_{x} e^{i p \cdot x} \mathscr{O}_{1}^{s_{1}}(x, t) \mathscr{O}_{2}^{s_{2}}(0,0)^{\dagger}\right\rangle \\
& =\sum_{n}\left\langle 0\left|\mathscr{O}_{1}^{s_{1}}\right| n\right\rangle\left\langle n\left|\mathscr{O}_{2}^{s_{2}}\right| 0\right\rangle e^{-E_{n}(p) t}
\end{aligned}
$$

The operators should have the same $J^{P}$ quantum numbers as the state of interest. Two typical $0^{+}$interpolating operators are,

$$
P(x)=\bar{u}(x)_{a}^{\alpha} \gamma_{5}^{\alpha \beta} d_{a}^{\beta}(x), \quad A_{t}(x)=\bar{u}_{a}^{\alpha}(x) \gamma_{t}^{\alpha \kappa} \gamma_{5}^{\kappa \beta} d_{a}^{\beta}(x)
$$

For our choice of source we can obtain the mass $\left(f_{0}\right)$ from a simultaneous fit with 5 parameters

\footnotetext{
${ }^{1}$ The gauge fixing was performed to an accuracy of $\Theta<10^{-14}$ using the FACG algorithm of [8]
} 
to the following 8 correlation functions,

$$
\begin{array}{ll}
C_{P P}^{W L}=f_{3} f_{1}\left(e^{-f_{0} t}+e^{-f_{0}\left(t-L_{t}\right)}\right), & C_{A_{t} A_{t}}^{W L}=f_{4} f_{2}\left(e^{-f_{0} t}+e^{-f_{0}\left(t-L_{t}\right)}\right), \\
C_{P A_{t}}^{W L}=f_{3} f_{2}\left(e^{-f_{0} t}-e^{-f_{0}\left(t-L_{t}\right)}\right), & C_{A_{t} P}^{W L}=f_{4} f_{1}\left(e^{-f_{0} t}-e^{-f_{0}\left(t-L_{t}\right)}\right), \\
C_{P P}^{W W}=f_{3} f_{3}\left(e^{-f_{0} t}+e^{-f_{0}\left(t-L_{t}\right)}\right), & C_{A_{t} A_{t}}^{W W}=f_{4} f_{4}\left(e^{-f_{0} t}+e^{-f_{0}\left(t-L_{t}\right)}\right), \\
C_{P A_{t}}^{W W}=f_{3} f_{4}\left(e^{-f_{0} t}-e^{-f_{0}\left(t-L_{t}\right)}\right), & C_{A_{t} P}^{W W}=f_{4} f_{3}\left(e^{-f_{0} t}-e^{-f_{0}\left(t-L_{t}\right)}\right) .
\end{array}
$$

Here $\mathrm{W}$ indicates Wall and L indicates Local sources and sinks. A Wall-Wall correlator is defined as the contraction of the sum of the propagators on the source and the sink timeslices. Using timereversal symmetry we "fold" our correlation functions about the midpoint $\frac{L_{t}}{2}$ taking care to use the correct sign for the sinh-behaving correlation functions.

The relations in Eq.2.3 then allow us to extract the pseudoscalar decay constant,

$$
f_{P}=Z_{A} \sqrt{\frac{2 f_{2}^{2}}{L^{3} f_{0}}} .
$$

Where $L$ is the spatial length of the lattice. Since the axial renormalisation constant $Z_{A}$ is known for these lattices only from lattice perturbation theory [10], we instead consider the ratio $\frac{f_{K}}{f_{\pi}}$ as a test of our methodology.

\subsection{Baryons}

A simple baryon interpolating operator (with different fermion fields $\psi, \chi$ and $\phi$ ) is,

$$
B_{i}^{\kappa}(x)=\varepsilon_{a b c}\left(\left(\psi_{a}^{\alpha}(x)\right)^{T} C \Gamma_{i}^{\alpha \beta} \chi_{b}^{\beta}(x)\right) \phi(x)_{c}^{\kappa},
$$

where $C=i \gamma_{y} \gamma_{t}$. We will consider $J^{P}=1 / 2^{+}$and $3 / 2^{+}$baryons. For baryons with a subscript index $i\left(\Gamma_{i}=\gamma_{i}\right)$ their correlation function is a combination of $J=1 / 2$ and $J=3 / 2$ and so their spin components should be projected out. For $\Gamma_{i}=\gamma_{5}$ no spin projection is necessary ${ }^{2}$. We save the correlation functions with open dirac indices and open spin indices and perform spin and parity projections as post-processing.

We select the positive parity for our correlation functions using the following projectors (acting on the open upper dirac indices) [11],

$$
L_{4 / 5}^{\alpha \beta}=\frac{1}{2}\left(I \pm \gamma_{t}\right)^{\alpha \beta}, \quad C_{i j}^{(L)}(p, t)=L^{\kappa^{\prime} \kappa} C_{i j}^{\kappa \kappa^{\prime}}(p, t) .
$$

We note that the backwards propagating $L_{5}$ projected correlator and the forwards propagating $L_{4}$ are somewhat statistically independent, which allows us to fold these two correlators into one another.

Following $[12,13,14]$ we use the spin projectors to extract the spin- $1 / 2$ and spin- $3 / 2$ components at zero momentum (acting on the lower spin indices),

$$
\begin{gathered}
P_{i j}^{1 / 2}=\frac{1}{3} \gamma_{i} \gamma_{j}, \quad P_{i j}^{3 / 2}=\delta_{i j}-\frac{1}{3} \gamma_{i} \gamma_{j} . \\
C_{(P)}^{\kappa K^{\prime}}(t)=P_{k j} C_{j k}^{\kappa K^{\prime}}(t) .
\end{gathered}
$$

After parity and spin projection, the resulting correlation functions are spatially averaged, bootstrapped and fit to a single exponential.

\footnotetext{
${ }^{2}$ In Figs. 1 and 2 baryons of this type will be labelled $B_{P}$.
} 


\section{Methodology}

For our measurement of the spectrum here and the work on a possible tetraquark candidate [15] we were required to contract relativistic fermions with non-relativistic fermions. The relativistic fermions use a modified (from the DDHMC package), deflated SAP solver [16] for the light, strange and charm quarks, this is a MPI code written in c. The NRQCD propagator code [6] is serial and written in Fortran. We decided that the best way to tackle forming correlation functions was to save our propagators and write a thread-parallel, SSE-vectorised, contraction library that can read in saved propagators and compute the required correlation functions.

This is a beneficial method for us because disk space is cheap and contractions are cheap, whereas inversions with the resources available to us are computationally expensive, especially for near-physical light quark masses. To reduce our disk space requirement, we save our propagators in single precision as the difference between correlation functions from single or double precision propagators is of the order of the single precision ULP and hence much lower than our statistical resolution.

For the Baryons we choose to compute the correlation functions with open Dirac indices, performing the parity projections (Eq.2.6) and spin projections (Eq.2.7) necessary as post-processing steps.

\subsection{Actions}

We use fully relativistic, dynamic, non-perturbatively tuned [17] Wilson-Clover $\mathrm{u} / \mathrm{d}$ and $\mathrm{s}$ quarks, with a partially quenched valence strange quark tuned to physical $\kappa_{s}$ [18].

We use a relativistic (Tsukuba) charm quark action [19],

$$
\begin{aligned}
M(x, y) & =\delta_{x, y}\left(1-\kappa c_{E} \sum_{i} F_{i 4} \sigma_{i 4}-\kappa c_{B} \sum_{i, j} F_{i j} \sigma_{i j}\right) \\
& -\kappa \sum_{\mu}\left(\left(r_{\mu}-v \gamma_{\mu}\right) U_{x, \mu} \delta_{x+\hat{\mu}, y}+\left(r_{\mu}+v \gamma_{\mu}\right) U_{x, \mu}^{\dagger} \delta_{x, y+\hat{\mu}}\right) .
\end{aligned}
$$

The tuning has already been performed for the ensembles we will be using in this work [19].

For the b-quarks we use the tree level tadpole-improved NRQCD hamiltonian [20, 21],

$$
\begin{aligned}
H & =-\frac{\Delta^{(2)}}{2 M_{0}}-c_{1} \frac{\left(\Delta^{(2)}\right)^{2}}{8 M_{0}^{3}}+\frac{c_{2}}{U_{0}^{4}} \frac{i g}{8 M_{0}^{2}}(\tilde{\Delta} \cdot \tilde{E}-\tilde{E} \cdot \tilde{\Delta}) \\
& -\frac{c_{3}}{U_{0}^{4}} \frac{g}{8 M_{0}^{2}} \sigma \cdot(\tilde{\Delta} \times \tilde{E}-\tilde{E} \times \tilde{\Delta})-\frac{c_{4}}{U_{0}^{4}} \frac{g}{2 M_{0}} \sigma \cdot \tilde{B} \\
& +c_{5} \frac{a^{2} \Delta^{(4)}}{24 M_{0}}-c_{6} \frac{a\left(\Delta^{(2)}\right)^{2}}{16 n M_{0}^{2}} .
\end{aligned}
$$

For the tadpole improvement $U_{0}$ we have tuned with the fourth root of the plaquette rather than the mean Landau link as it has lower statistical variance and does not suffer from the Gribov ambiguity. $M_{0}$ is a free parameter we must tune. 
We have retuned the NRQCD action ${ }^{3}$ (using point sources) for these ensembles by fitting linearly in the lattice momentum $\tilde{p}^{2}$ to,

$$
a E(p)=a M_{0}+\frac{\tilde{p}^{2}}{2 a M_{\mathrm{ph}}}, \quad \tilde{p}_{\mu}=a \sin \left(\frac{2 \pi n_{\mu}}{L_{\mu}}\right) .
$$

The free parameter $M_{0}$ is varied until $M_{\text {ph }}$ gives the physical $\Upsilon=9.4 \mathrm{GeV}$. For the extraction of masses of particles with NRQCD quarks there is an additive mass renormalisation that must be included; when one considers mass differences, say of spin- $1 / 2$ to spin-3/2 baryons, this factor cancels.

\section{Results}

\subsection{Ensemble properties}

Some basic ensemble properties are listed in Tab.1. Using the heavily-constrained fit in Eq.2.3 and multiple time sources we were able to measure the pion mass to around a $6 \times$ higher precision than the original analysis of this ensemble [1] and greater than $3 \times$ statistical resolution for the pion masses of $E_{H}$ and $E_{M}$. These results and propagators will also be used in the determination of the binding of a possible tetraquark candidate we have presented at this conference $[22,15]$.

\begin{tabular}{c|ccc}
\hline Label & $E_{H}$ & $E_{M}$ & $E_{L}$ \\
\hline$a^{-1}[\mathrm{GeV}][7]$ & $2.194(10)$ & $2.194(10)$ & $2.194(10)$ \\
$\kappa_{l}$ & 0.13754 & 0.13770 & 0.13781 \\
$\kappa_{s}$ & 0.13666 & 0.13666 & 0.13666 \\
$a m_{\pi}$ & $0.18928(36)$ & $0.13618(46)$ & $0.07459(54)$ \\
$a m_{K}$ & $0.27198(28)$ & $0.25157(30)$ & $0.23288(25)$ \\
$f_{K} / f_{\pi}$ & $1.0827(14)$ & $1.1151(33)$ & $1.2012(75)$ \\
$M_{\Upsilon}[\mathrm{GeV}]$ & $9.477(79)$ & $9.438(71)$ & $9.392(76)$ \\
Configurations (Measurements) & $400(800)$ & $800(800)$ & $195(3078)$ \\
\hline
\end{tabular}

Table 1: Overview of our ensemble parameters. Fit ranges were chosen so that $\chi^{2} /$ d.o.f was close to 1 . Inversions on multiple time-slices were used for $E_{H}$ and $E_{L}$. All configurations have extent $32^{3} \times 64$.

For $\approx 1 \%$ of inversions the light-quark propagator on $E_{L}$ converged but the pseudoscalar correlation function did not decay exponentially. Such configurations we believe to be unphysical labelling them as exceptional and discard them.

\subsection{Splittings}

We illustrate our results for singly, doubly and triply heavy baryon masses in Fig.1, we compare to the work of [3] and to experiment where available. While we are at fixed lattice spacing and the work of [3] is extrapolated to $a^{2} \rightarrow 0$, we still see good agreement, implying that cut off effects are under control. Our chiral extrapolations are obtained via quadratic fits to the data (represented in the figure by open symbols, with the leftmost being the result for $E_{L}$ and the rightmost the result for $E_{H}$ ) in $m_{\pi}^{2}$, with a preliminary evaluation of the result in the chiral limit as a filled symbol.

\footnotetext{
${ }^{3}$ Compared to previous works using these configurations [6]
} 


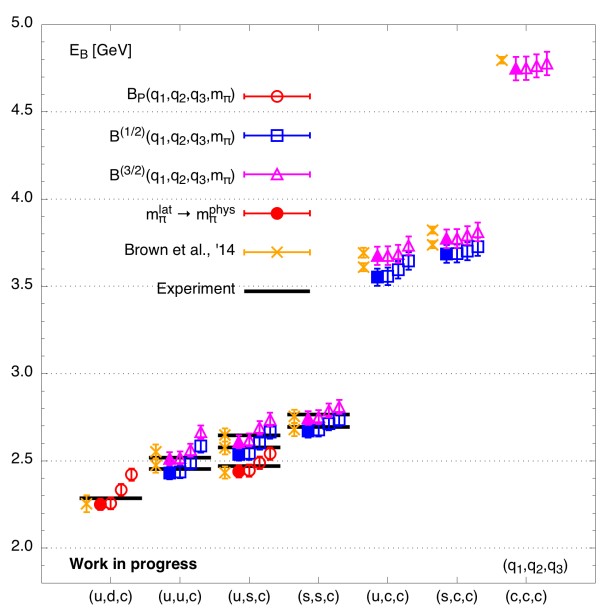

(a) $b=0, c \geq 1$

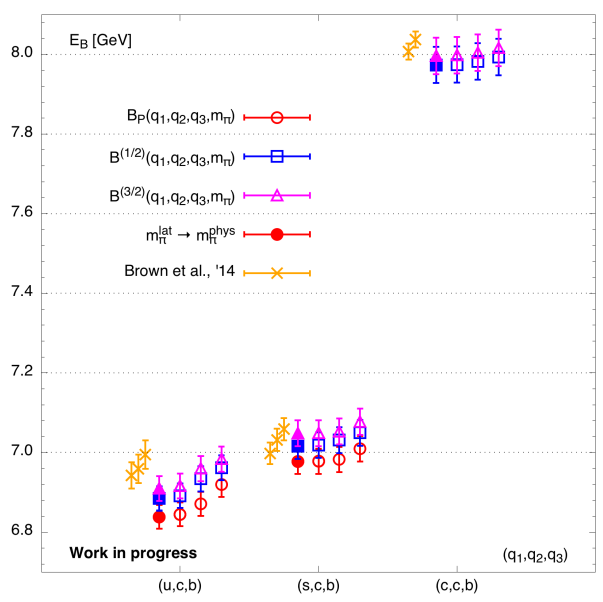

(c) $b=1, c \geq 1$

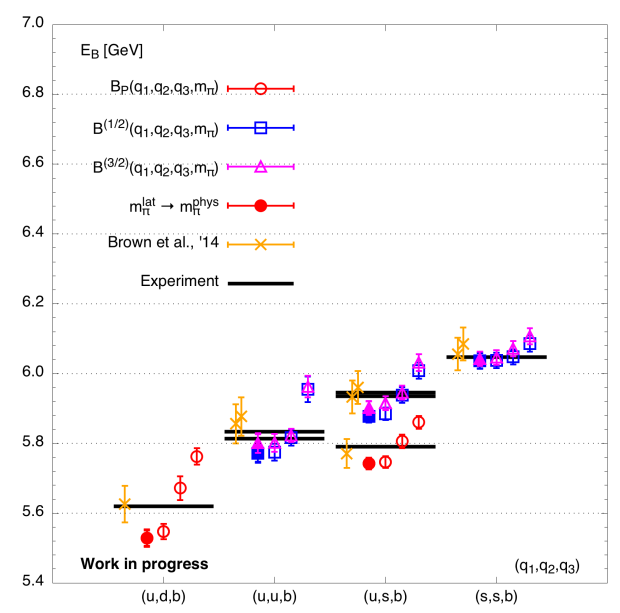

(b) $b=1, c=0$

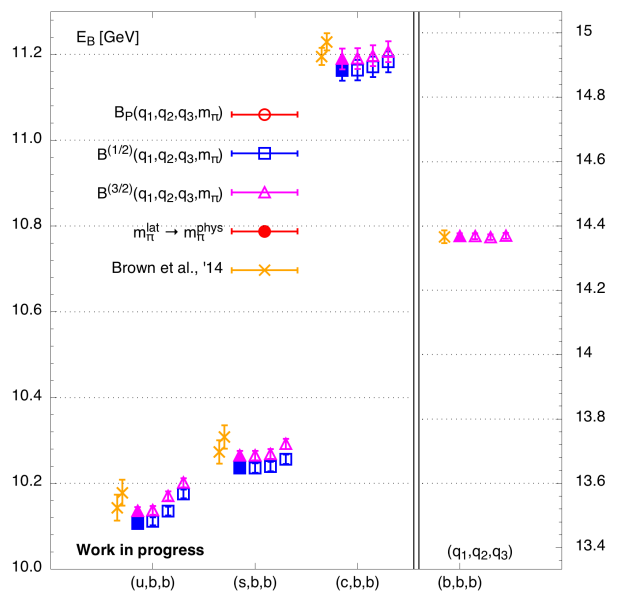

(d) $b>1, c \geq 0$

Figure 1: Masses for baryons containing at least one heavy $c$ or $b$ quark, shown alongside the results of [3] and experimental results where possible. Open symbols indicate our measurements, filled symbols are our extrapolation to the physical pion.

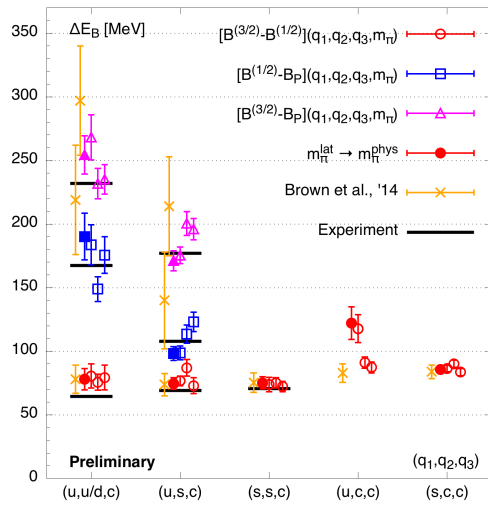

(a) $b=0$ baryon splittings.

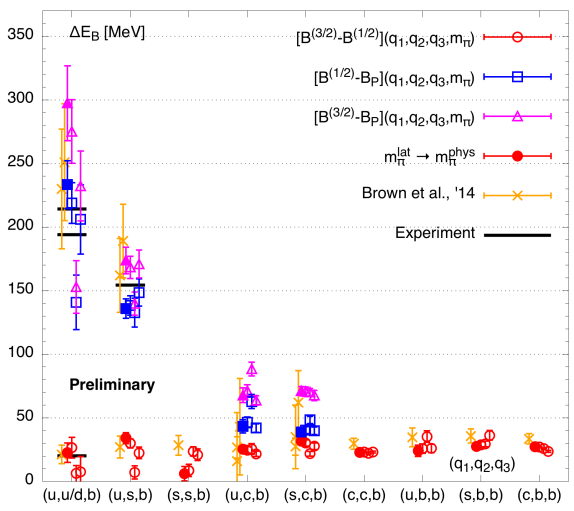

(b) $b>0$ baryon splittings.

Figure 2: Charm and bottom baryon mass splittings between $J=3 / 2^{+}$and $J=1 / 2^{+}$. The results of [3] are also shown as are the experimental determinations of these quantities where they exist. 
Fig. 2 shows the splittings of $J=3 / 2^{+}$to $J=1 / 2^{+}$channels where the labels $B^{(3 / 2)}$ and $B^{(1 / 2)}$ indicate the spin-projected $J=3 / 2^{+}$and $J=1 / 2^{+}$baryon masses respectively (with $\Gamma_{i}=\gamma_{i}$ in Eq. 2.5). $B_{p}$ indicates $J=1 / 2^{+}$baryon masses, this time with $\Gamma_{i}=\gamma_{5}$. In these figures $q_{i}$ indicates the quark flavour content. We observe agreement within errors with [3] and significant error reduction in some of the channels, particularly those with a single light quark or strange quark. Results are preliminary and the analysis is ongoing.

\section{Conclusions}

We have tested our contraction software on charm and bottom baryons, and some parts of the simple meson spectrum. We find consistent results with experiment where results exist and agreement within errors with another study that used a different lattice action. With the benefit of variance reduction from gauge fixed wall sources, well-tuned NRQCD bottom quarks and relativistic charm quarks and a large cache of saved propagators, fast and accurate spectroscopic calculations are possible.

\section{Acknowledgements}

RJH, AF, RL and KM are supported by NSERC of Canada. The authors would like to thank M. Wurtz for initial help with the initial NRQCD portion of the project. Both light and heavy quark propagators have been produced and saved on Compute Canada's GPC machine at SciNet.

\section{References}

[1] PACS-CS collaboration, S. Aoki et al.Phys. Rev. D79 (2009) 034503.

[2] M. G. Beckett et al.Comput. Phys. Commun. 182 (2011) 1208-1214.

[3] Z. S. Brown et al.Phys. Rev. D90 (2014) 094507.

[4] R. Lewis et al.Phys. Rev. D64 (2001) 094509.

[5] N. Mathur et al.Phys. Rev. D66 (2002) 014502.

[6] R. Lewis and R. M. WoloshynPhys. Rev. D79 (2009) 014502.

[7] PACS-CS collaboration, Y. Namekawa et al.Phys. Rev. D87 (2013) 094512.

[8] RBC, UKQCD collaboration, R. J. HudspithComput. Phys. Commun. 187 (2015) 115-119.

[9] D. Daniel et al.Phys. Rev. D46 (1992) 3130-3145.

[10] S. Aoki et al.Phys. Rev. D58 (1998) 074505, [hep-lat/9802034].

[11] W. Melnitchouk et al.Phys. Rev. D67 (2003) 114506, [hep-lat/ 0202022 ].

[12] P. Van NieuwenhuizenPhys. Rept. 68 (1981) 189-398.

[13] M. Benmerrouche et al.Phys. Rev. C39 (1989) 2339-2348.

[14] UKQCD collaboration, K. C. Bowler et al.Phys. Rev. D54 (1996) 3619-3633.

[15] A. Francis et al. 1607.05214.

[16] M. LuscherComput. Phys. Commun. 165 (2005) 199-220.

[17] JLQCD, CP-PACS collaboration, S. Aoki et al.Phys. Rev. D73 (2006) 034501.

[18] C. Lang et al.Phys. Rev. D90 (2014) 034510.

[19] PACS-CS collaboration, Y. Namekawa et al.Phys. Rev. D84 (2011) 074505.

[20] C. T. H. Davies et al.Phys. Rev. D50 (1994) 6963-6977.

[21] B. A. Thacker and G. P. LepagePhys. Rev. D43 (1991) 196-208.

[22] A. Francis, R. J. Hudspith, R. Lewis and K. Maltman, Pos lattice, 132, 2016. 